\title{
Unusual acute lupus hemophagocytic syndrome - a test of diagnostic criteria: a case report
}

\author{
Wijetunga Mudalige Udai Akalanka Wijetunga ${ }^{1 *}$, Ravindra Laxman Satarasinghe², \\ Balasuriya Mudiyanselage Dayananda ${ }^{1}$ and Ganhewage Kokila Darshani ${ }^{1}$
}

\begin{abstract}
Background: Hemophagocytic lymphohistiocytosis is an aggressive life-threatening syndrome of excessive immune activation. Hemophagocytic lymphohistiocytosis due to systemic lupus erythematosus is described as acute lupus hemophagocytic syndrome. Acute lupus hemophagocytic syndrome presenting with negative antinuclear antibody is uncommon.

Case presentation: A 57-year-old Sri Lankan woman presented with intermittent fever, weight loss, episodic confusion, and alopecia for 3 months. Investigations showed pancytopenia. Her erythrocyte sedimentation rate was $76 \mathrm{~mm} /$ hour and C-reactive protein was $2 \mathrm{mg} / \mathrm{l}$. Septic screen was negative except for a positive sputum culture for Gram-negative coliforms. Chest X-ray was normal. Direct antiglobulin test was positive. Fever persisted with clinical worsening despite treatment with intravenous antibiotics. Antinuclear antibodies and doublestranded-deoxyribonucleic acid antibodies were negative. Bone marrow aspiration revealed features compatible with hemophagocytosis. Her serum ferritin and triglycerides were elevated. Diagnosis of hemophagocytic lymphohistiocytosis due to an evolving autoimmune disorder was made and she was treated with steroids. She showed a dramatic improvement and was discharged on oral steroids.

After 6 months, while the steroids were being tapered she experienced oral ulcers, frothy urine, and ankle swelling and she was rehospitalized. Urine analysis revealed proteinuria with active sediment. Antinuclear antibodies and doublestranded-deoxyribonucleic acid antibodies were positive. Complement C3 and C4 were reduced. A renal biopsy revealed class IV-G lupus nephritis with immunofluorescence pattern consistent with systemic lupus erythematosus. Steroid dose was increased and mycophenolate mofetil was commenced. She improved.
\end{abstract}

Conclusions: This case showcases an uncommon presentation of acute lupus hemophagocytic syndrome with initial negative antinuclear antibody probably due to its cytokine-mediated pathogenesis. This is the first such reported case in South Asia to the best of our knowledge. According to the American College of Rheumatology criteria, our patient did not fulfill the criteria for systemic lupus erythematosus diagnosis for the initial hospitalization. But, according to the 2012 Systemic Lupus International Collaborating Clinics criteria, she did fulfill the criteria for systemic lupus erythematosus even in the first hospitalization which was subsequently proven with renal biopsy findings. This case confirms the increased sensitivity of Systemic Lupus International Collaborating Clinics criteria over American College of Rheumatology criteria in diagnosis of systemic lupus erythematosus.

Keywords: Systemic lupus erythematosus, Acute lupus hemophagocytic syndrome, Hemophagocytic lymphohistiocytosis, Case report

\footnotetext{
* Correspondence: udaiwijetunga@gmail.com

${ }^{1}$ Postgraduate Institute of Medicine, University of Colombo, Colombo, Sri

Lanka

Full list of author information is available at the end of the article
} 


\section{Background}

Hemophagocytic lymphohistiocytosis (HLH) is an aggressive and life-threatening syndrome of excessive immune activation characterized by increased proliferation and activation of benign macrophages with hemophagocytosis in the bone marrow and other reticuloendothelial systems $[1,2]$. HLH is also known to cause proliferation and activation of $\mathrm{T}$ lymphocytes and macrophages producing an excessive inflammatory response and hypersecretion of cytokines [3]. HLH is a rare disease in adults and the incidence is one patient per million persons per year [1]. HLH can occur as a familial primary form or secondary to infections, rheumatologic diseases, medications, and neoplasms [3]. Reactive hemophagocytosis occurring due to underlying active systemic lupus erythematosus (SLE) is described as acute lupus hemophagocytic syndrome (ALHS) [4].

ALHS is usually associated with high titers of antinuclear antibodies (ANAs) and hypocomplementemia, suggesting immune complex-mediated mechanisms in the pathogenesis of ALHS [5]. The incidence of autoimmuneassociated hemophagocytic syndrome in SLE was found to be $4.6 \%$ in one study [6].

The usual patient presentation of HLH is characterized by prolonged fever, weight loss, hepatosplenomegaly, lymphadenopathy, coagulopathy, pancytopenia, liver function abnormalities, hyperferritinemia, and hypertriglyceridemia $[2,3]$. It is sometimes difficult to differentiate an acute SLE case from HLH due to them having many common features. To make matters worse, it is not easy to differentiate hemophagocytic syndromes occurring secondary to infection from those occurring due to a rheumatological cause like SLE. It is essential that this distinction is made because HLH due to SLE responds rapidly to steroids and immunosuppressants [1].

\section{Case presentation}

A 57-year-old previously healthy Sri Lankan woman presented to our hospital with 3 months' history of intermittent fever, anorexia, and weight loss. She had lost 8 $\mathrm{kg}$ over this period. A worsening of the fever characterized by daily episodes with spikes prompted admission. She complained of significant hair loss and on and off confusional episodes over the last 2 weeks, but denied symptoms of focal infections including respiratory symptoms. Also, she disaffirmed any history of arthritis and features of autoimmune diseases such as photosensitive rashes, oral ulcers, or symptoms of Raynaud's phenomenon. On examination her body mass index was $16 \mathrm{~kg} / \mathrm{m}^{2}$. She was febrile with a temperature of $37.8{ }^{\circ} \mathrm{C}$ $\left(100^{\circ} \mathrm{F}\right)$, pale, and non-scarring alopecia was evident. Her other vital parameters were normal (blood pressure 130/ $80 \mathrm{mmHg}$, pulse rate $92 /$ minute, respiratory rate
16 breaths/minute). The rest of the examination was unremarkable.

A full blood count done a month before admission revealed a bicytopenia with leukopenia and thrombocytopenia. Her erythrocyte sedimentation rate (ESR) was 70 $\mathrm{mm}$ /hour and C-reactive protein (CRP) was $6 \mathrm{mg} / \mathrm{l}$ at that time. The differential diagnoses considered at this point were infections such as tuberculosis (TB), infective endocarditis, hematological and solid organ malignancies, and autoimmune diseases.

Initial full blood count after admission showed a pancytopenia which was confirmed on blood picture: white

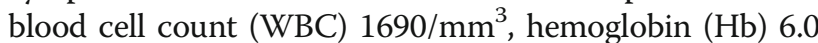
$\mathrm{g} / \mathrm{dl}$, and platelets $102,000 / \mathrm{mm}^{3}$. Her ESR was $76 \mathrm{~mm} /$ hour and CRP was $2 \mathrm{mg} / \mathrm{l}$. A sputum culture was positive for Gram-negative coliforms. A chest X-ray was normal. Intravenously administered antibiotics were started according to the antibiotic sensitivity tests (ABSTs) but her fever continued. Repeated blood and urine cultures were negative. A urine full report was normal. A diagnosis of a respiratory tract infection was unlikely because she did not have any respiratory tract symptoms, examination of her lungs was normal, her CRP was normal repeatedly, and her fever did not subside despite continuing apparently sensitive intravenously administered antibiotics with no clinical improvement.

A two-dimensional echocardiogram revealed no evidence of infective endocarditis. Sputum for acid-fast bacilli and Mantoux test were negative. Bone marrow aspiration was done and it revealed hemophagocytic activity in her bone marrow suggestive of primary or secondary hemophagocytic syndrome. There were no features of hematological malignancy in the bone marrow (Figs. 1, 2, 3 and 4). Serum ferritin was highly elevated (>1650 ng/dl). Serum lactate dehydrogenase (LDH) was elevated (926 U/l). Serum triglyceride level was $270 \mathrm{mg} / \mathrm{dl}$. Serum protein

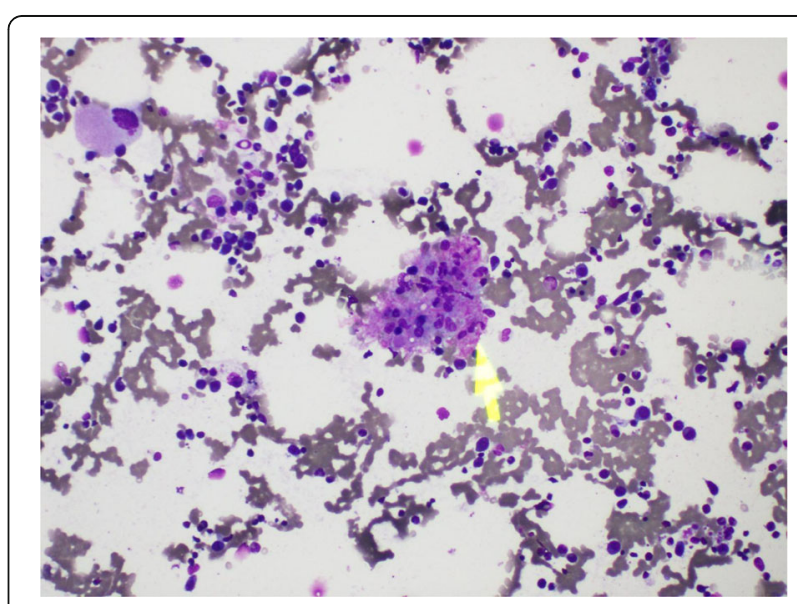

Fig. 1 Bone marrow biopsy (x20 magnification) : Phagocytosis of RBC and WBC are seen (arrow) 


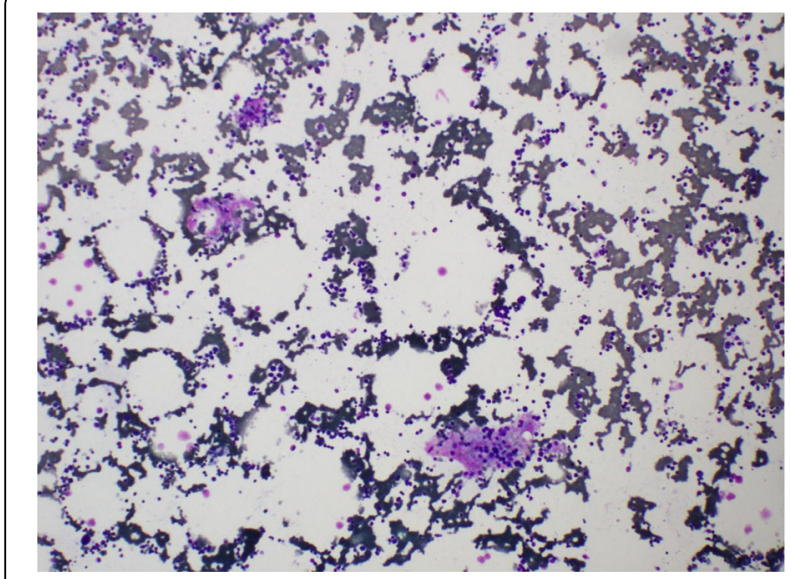

Fig. 2 Bone marrow biopsy (x4 magnification) : Multiple phagocytic foci are seen electrophoresis showed only a polyclonal gammopathy, thus excluding the presence of a monoclonal band. Carcinoembryonic antigen (CEA) level was normal.

Epstein-Barr virus (EBV), cytomegalovirus (CMV), and rubella virus antibodies were negative. Screening for hepatitis $B$, hepatitis $C$, and human immunodeficiency virus (HIV) was negative. Bone marrow for TB polymerase chain reaction (PCR) and culture were negative. An ultrasound scan of her abdomen and pelvis was normal.

An autoimmune screen was negative with ANA, antidouble-stranded-deoxyribonucleic acid (dsDNA), rheumatoid factor, cytoplasmic antineutrophil cytoplasmic antibody (c-ANCA), and perinuclear antineutrophil cytoplasmic antibody (p-ANCA) being negative. A direct antiglobulin test was positive with specificity to immunoglobulin A (IgA).

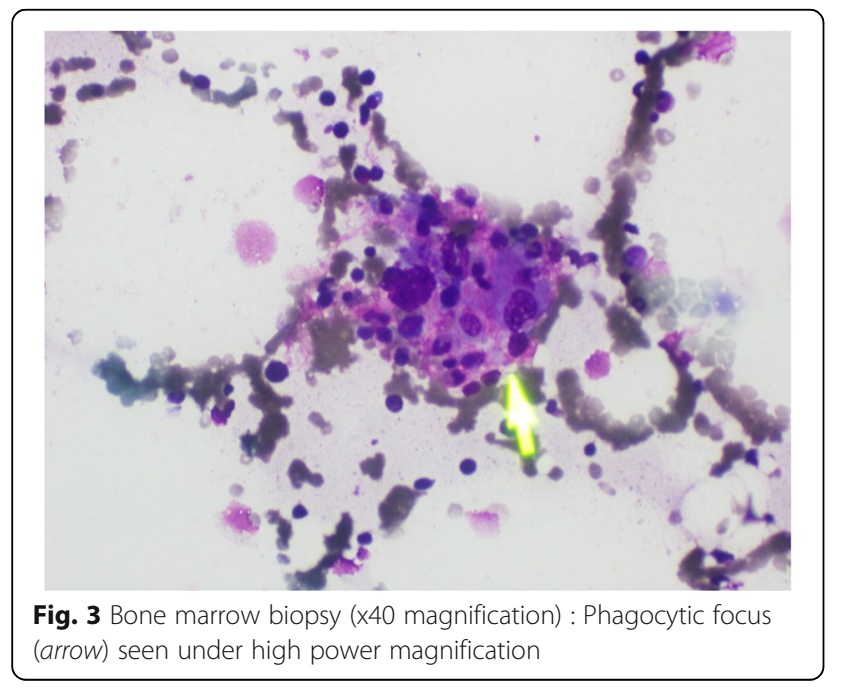

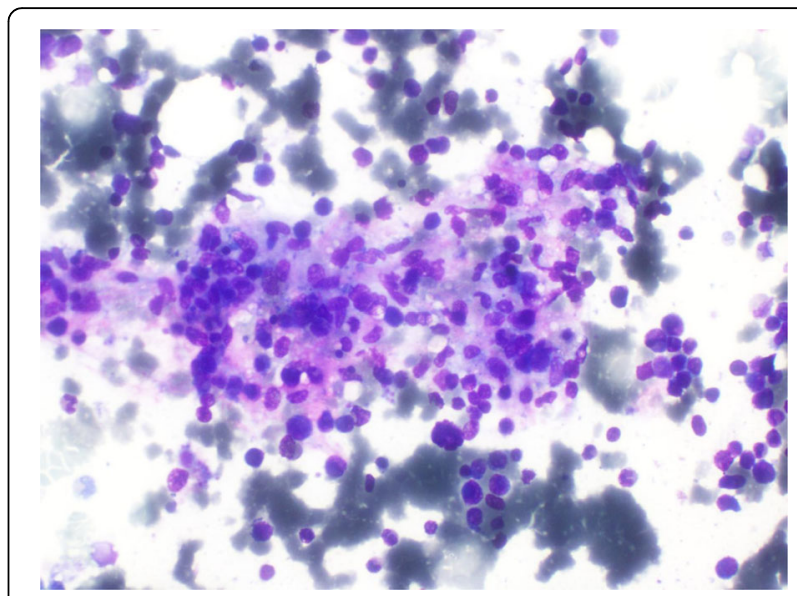

Fig. 4 Bone marrow biopsy (x40 magnification) : Phagocytic focus with increased number of phagocytosed cells seen under high power magnification

Due to her persistent fever, a diagnosis of hemophagocytic syndrome secondary to an evolving autoimmune disease was made and she was started on intravenously administered methylprednisolone pulses. She was given intravenously administered methylprednisolone $1 \mathrm{~g}$ daily for 3 days which was followed by orally administered prednisolone $60 \mathrm{mg}$ daily. She showed a dramatic response with clinical improvement, resolution of fever, resolution of pancytopenia, reduction in LDH and ferritin, and reduction in ESR. She was discharged and was sent with a tapering dose of orally administered prednisolone. On follow-up, she reported no adverse effects of medication and she was compliant with her treatment regime.

After 6 months while on a tapering dose of prednisolone, she experienced intermittent fever, oral ulcers, frothy urine, and ankle swelling for 3 weeks which motivated a second admission. An evaluation revealed subnephrotic range proteinuria with active sediment on urine analysis. ANA (>1/80) and anti-dsDNA (>1/10) were positive. Complement C3 and C4. $(74 \mathrm{mg} / \mathrm{dl}$ and $2.9 \mathrm{mg} / \mathrm{dl})$ were reduced. A renal biopsy was done and it revealed class IV-G lupus nephritis with immunofluorescence pattern consistent with SLE. A diagnosis of SLE with renal involvement was made. A summary of investigation results are given in Table 1.

She was started on orally administered prednisolone $60 \mathrm{mg}$ daily and mycophenolate mofetil with good response. On follow-up she was clinically improved and has remained asymptomatic to date (9 months after discharge from hospital) while on immunosuppressive treatment. The prednisolone dose was gradually reduced and maintained at a dose of $7.5 \mathrm{mg}$ daily. Mycophenolate mofetil was continued. Thus with this new irrevocable evidence of SLE, the diagnosis of the previous admission was revised as ALHS (Fig. 5). 
Table 1 Investigation summary

\begin{tabular}{|c|c|c|}
\hline & First admission & Second admission \\
\hline White blood cells & $1690 / \mathrm{mm}^{3}$ & $4900 / \mathrm{mm}^{3}$ \\
\hline Lymphocyte count & $740 / \mathrm{mm}^{3}$ & $420 / \mathrm{mm}^{3}$ \\
\hline Neutrophil count & $910 / \mathrm{mm}^{3}$ & $4210 / \mathrm{mm}^{3}$ \\
\hline Hemoglobin & $6.0 \mathrm{~g} / \mathrm{dl}$ & $8.4 \mathrm{~g} / \mathrm{dl}$ \\
\hline Platelets & $102,000 / \mathrm{mm}^{3}$ & $260,000 / \mathrm{mm}^{3}$ \\
\hline ESR & $76 \mathrm{~mm} /$ hour & 120 mm/hour \\
\hline CRP & $2 \mathrm{mg} / \mathrm{dl}$ & $<6 \mathrm{mg} / \mathrm{dl}$ \\
\hline LDH & $926 \mathrm{u} / \mathrm{l}$ & $482 \mathrm{u} / \mathrm{l}$ \\
\hline Ferritin & $>1650 \mathrm{ng} / \mathrm{ml}$ & $>1650 \mathrm{ng} / \mathrm{ml}$ \\
\hline AST & $106 \mathrm{u} / \mathrm{l}$ & $105 \mathrm{u} / \mathrm{l}$ \\
\hline ALT & $64 \mathrm{u} / \mathrm{l}$ & $97 \mathrm{u} / \mathrm{l}$ \\
\hline ALP & $85 \mathrm{iu} / \mathrm{l}$ & $166 \mathrm{iu} / \mathrm{l}$ \\
\hline Serum bilirubin & $0.4 \mathrm{mg} / \mathrm{dl}$ & $0.3 \mathrm{mg} / \mathrm{dl}$ \\
\hline Serum protein & $5.4 \mathrm{~g} / \mathrm{dl}$ & $6.4 \mathrm{~g} / \mathrm{dl}$ \\
\hline Serum albumin & $3.0 \mathrm{~g} / \mathrm{dl}$ & $3.8 \mathrm{~g} / \mathrm{dl}$ \\
\hline Serum sodium & $131 \mathrm{mEq} / \mathrm{l}$ & $140 \mathrm{mEq} / \mathrm{l}$ \\
\hline Serum potassium & $4.6 \mathrm{mEq} / \mathrm{l}$ & $4.1 \mathrm{mEq} / \mathrm{l}$ \\
\hline Serum creatinine & 89 micromol/l & 88 micromol/l \\
\hline
\end{tabular}

ALP alkaline phosphatase, $A L T$ alanine aminotransferase, $A S T$ aspartate aminotransferase, CRP C-reactive protein, ESR erythrocyte sedimentation rate, $L D H$ lactate dehydrogenase

\section{Discussion}

An updated set of diagnostic criteria for HLH was proposed in 2004 by The Histiocyte Society. The diagnosis needs either a molecular diagnosis consistent with HLH including the identification of pathologic mutations of PRF1, UNC13D, or STX11 or the presence of at least five out of eight features: fever, splenomegaly, bi/pancytopenia, hypertriglyceridemia or hypofibrinogenemia, ferritin $>500 \mathrm{ng} / \mathrm{ml}$, hemophagocytosis in the bone marrow/lymph nodes/spleen, low natural killer cell activity, soluble CD25 (soluble interleukin-2 receptor) $>2400 \mathrm{U} / \mathrm{ml}[7,8]$.

This patient's initial presentation fulfilled the criteria for HLH according to the above criteria although the underlying cause was not clear at the time. The combination of suggestive bone marrow results, elevated serum triglycerides and ferritin, and fever and pancytopenia completed the diagnostic criteria for HLH in this patient.

The main underlying causes for HLH are autoimmunity, infection, and malignancy [9]. The signs of an evolving autoimmune disease were evident even at the initial presentation. They included persistent fever and symptoms despite treating with intravenously administered antibiotics, presence of significant alopecia, direct antiglobulin test being positive and rapid improvement after treatment with steroids. She did not test positive for ANA or dsDNA tests initially and did not fulfill the diagnostic criteria for SLE according to the 1982 American College of Rheumatology (ACR) criteria or its 1997 update. Thus the diagnosis was initially given as HLH probably due to an underlying evolving autoimmune disease.

Of interest, according to the 1982 ACR criteria and even its 1997 update, our patient did not fulfil the criteria for SLE diagnosis for the initial hospitalization. These two classifications need at least four out of 11 criteria to be present for a diagnosis of SLE to be made. But this patient only fulfilled two criteria at best. According to the 2012 Systemic Lupus International Collaborating Clinics (SLICC) revised ACR SLE classification criteria [10], our patient fulfilled the criteria for SLE even in the first hospitalization. She had four of the diagnostic criteria including non-scarring alopecia, leukopenia/lymphopenia, history of acute confusional states, and positive direct antiglobulin test. A fifth criterion was almost fulfilled with a platelet count of $102,000 / \mathrm{mm}^{3}$ being just above $100,000 /$ $\mathrm{mm}^{3}$. Thus the diagnosis of ALHS is further confirmed.

Several days after our patient's admission, it was clear to us that her fever was not due to an infection although the sputum culture turned out positive for coliforms. Coliforms are well recognized to be isolated in sputum cultures in hospitalized patients where they colonize the upper respiratory tracts, especially in patients receiving long-term antibiotics as in the case of our patient. In the absence of other signs of infection coliforms isolates should be regarded as saprophytes in such clinical scenarios [11-13]. The likelihood of isolating an upper respiratory tract-colonizing organism is further increased in this patient especially because of the absence of a productive cough. Thus, she may have given a suboptimal specimen mostly consisting of saliva and upper respiratory tract secretions, leading to isolation of a coliform growth in the sputum culture. It was further confirmed when treatment with sensitive antibiotics failed to resolve her fever. Repeatedly sterile blood cultures, persistently normal CRP, absence of any respiratory symptoms or signs, and normal chest X-rays made the diagnosis of a respiratory tract infection unlikely.

Although the possibility of an infection is low in this patient, infections are known to trigger the onset of SLE $[14,15]$. Infections are responsible for significant morbidity and mortality in patients with SLE [16]. Patients with SLE have been found to be susceptible to pneumonia, urinary tract infections, and bacteremia without an identified focus [17]. This could be due to intrinsically associated immune deficiency or due to drugs used in the treatment of SLE [18].

Malignancies associated with HLH include malignant lymphoma, leukemias, and Waldenström's macroglobulinemia [9]. These were considered very unlikely due to unsuggestive bone marrow examination and blood 
Initial presentation with fever, loss of appetite and loss of weight. ANA, Ds DNA negative. Diagnosis of

'HLH probably secondary due to an underlying autoimmune disorder' made.

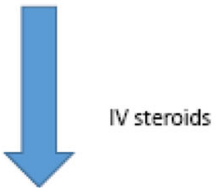

Resolution of fever and symptoms and discharged from hospital on oral steroids

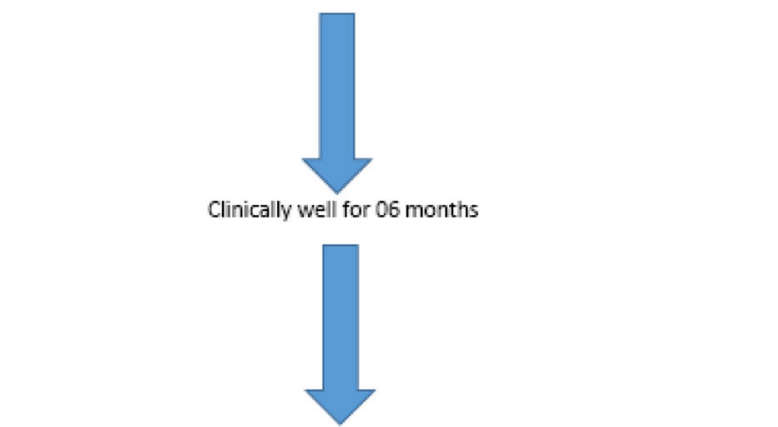

Relapse with oral ulcers, proteinuria and ankle swelling. Strongly positive ANA and Ds DNA with low complement.

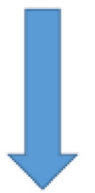

ALHS diagnosis made and treatment with Mycophenolate Mofetil started. Steroid dose increased.

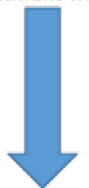

Complete recovery.

Fig. 5 ALHS acute lupus hemophagocytic syndrome, ANA antinuclear antibody, Ds DNA double-stranded-deoxyribonucleic acid, HLH hemophagocytic lymphohistiocytosis, $N$ intravenous

picture. These diagnoses were further excluded by a normal chest X-ray and ultrasound scan of her abdomen and by exclusion of a monoclonal band in the serum protein electrophoresis.

The occurrence of ALHS is usually seen in the setting of high ANA levels and hypocomplementemia indicating immune complex-mediated mechanisms in its pathogenesis [5]. But this patient had negative ANA in the initial presentation leading to masking of the underlying disease. Similar cases of ALHS with low titers of ANA have been reported and those authors have proposed a cytokine-mediated activation of histiocytes rather than an immune complexmediated process [5]. Thus it is most likely that our patient's initial disease presentation with HLH with a negative ANA was generated by cytokine-mediated mechanisms.

Most reported cases of ALHS have had positive and mostly high titers of ANA on presentation, but our patient interestingly presented with a negative ANA test and did not satisfy the diagnostic criteria for SLE according to the 1982 ACR criteria or its 1997 update, although she did fulfill the criteria for SLE when the 2012 SLICC revised ACR SLE classification criteria were considered. Her dramatic and persistent response to steroids strongly suggested an autoimmune etiology even when autoimmune markers were negative.

During her subsequent hospitalization, the evolving autoimmunity further revealed itself with strongly positive ANA and anti-dsDNA antibodies and the diagnosis of SLE was confirmed with positive renal biopsy findings. In retrospect, her autoimmunity must have responded to the steroids initially, but when the dose was reduced it flared up and it revealed itself with strongly positive ANA and dsDNA. Thus this case highlights an unusual presentation of ALHS with diagnostic difficulty probably due to its cytokine-mediated underlying pathogenesis. Due to its unusual presentation, the diagnosis of ALHS could only be made in retrospect with the evolution of the disease process. 


\section{Conclusions}

This case is interesting for many reasons. First, the initial presentation of ALHS with negative ANA and dsDNA is uncommon. Second, the initial presentation fulfils the diagnostic criteria for SLE according to the 2012 SLICC revised ACR SLE classification criteria but not with the 1982 ACR criteria or its 1997 update. Thus this case further indicates the sensitivity of the 2012 SLICC revised ACR SLE classification criteria. Third, this is the first such unusual case of ALHS reported in Sri Lanka and South Asia to the best of our knowledge.

Thus this case highlights the importance of having a high index of suspicion and the need to have close follow-up to detect ALHS when a patient presents with a picture of HLH, even when autoimmune markers are negative and the patient does not fulfill all the criteria for the diagnosis of SLE initially.

\section{Abbreviations}

ABST: Antibiotic sensitivity test; ACR: American College of Rheumatology; ALHS: Acute lupus hemophagocytic syndrome; ANA: Antinuclear antibody; cANCA: Cytoplasmic antineutrophil cytoplasmic antibody; CEA: Carcinoembryonic antigen; CMV: Cytomegalovirus; CRP: C-reactive protein; dsDNA: Double-stranded-deoxyribonucleic acid; EBV: Epstein-Barr virus; ESR: Erythrocyte sedimentation rate; Hb: Hemoglobin; HLH: Hemophagocytic lymphohistiocytosis; IgA: Immunoglobulin A LDH: Lactate dehydrogenase; p-ANCA: Perinuclear antineutrophil cytoplasmic antibody; PCR: Polymerase chain reaction; SLE: Systemic lupus erythematosus; SLICC: Systemic Lupus International Collaborating Clinics; TB: Tuberculosis; WBC: White blood cell count

\section{Acknowledgements}

Not applicable.

\section{Funding}

Not applicable.

\section{Availability of data and materials}

Data and materials are available on the patient's bed-head ticket, which is stored in the medical records office at Sri Jayawardenapura General Hospital, Sri Lanka.

\section{Authors' contributions}

WMUAW was the main author responsible for the manuscript and collected the data and wrote the manuscript. RLS read and corrected the manuscript and gave expert advice on management of the patient. BMD helped in writing the manuscript and collecting data. GKD helped in writing the manuscript. All authors read and approved the final manuscript.

\section{Authors' information}

WMUAW and BMD are registrars in Internal Medicine attached to the Postgraduate Institute of Medicine, University of Colombo, Sri Lanka. RLS is a visiting professor of Medicine at the Postgraduate Institute of Medicine, University of Colombo, Sri Lanka and a consultant physician at the Sri Jayawardenapura General Hospital, Sri Lanka. GKD is a senior registrar in Internal Medicine attached to the Postgraduate Institute of Medicine, University of Colombo, Sri Lanka.

\section{Competing interests}

The authors declare that they have no competing interests.

\section{Consent for publication}

Written informed consent was obtained from the patient for publication of this case report and any accompanying images. A copy of the written consent is available for review by the Editor-in-Chief of this journal.
Ethics approval and consent to participate

Ethical approval not taken as not applicable.

\section{Publisher's Note}

Springer Nature remains neutral with regard to jurisdictional claims in published maps and institutional affiliations.

\section{Author details}

${ }^{1}$ Postgraduate Institute of Medicine, University of Colombo, Colombo, Sri Lanka. ${ }^{2}$ Sri Jayawardenapura General Hospital, Thalapathpitiya, Nugegoda, Sri Lanka.

Received: 25 December 2016 Accepted: 30 May 2017

Published online: 07 July 2017

\section{References}

1. Rahal AK, Fernandez J, Dakhil C. Undiagnosed Systemic Lupus Erythematosus Presenting as Hemophagocytic Lymphohistiocytosis. Case Rep Rheumatol. 2015;2015:748713. Available from: http://www.ncbi.nlm.nih. gov/pubmed/26236531. Accessed 11 Oct 2016.

2. Botelho C, Ferrer F, Francisco L, Maia P, Mendes T, Carreira A. Acute lupus hemophagocytic syndrome: report of a case. Nefrol publicación Of la Soc Española Nefrol. 2010;30(2):247-51. Available from: http://www.ncbi.nlm.nih. gov/pubmed/20038974. Accessed 11 Oct 2016.

3. Henter J-I, Horne A, Aricó M, Egeler RM, Filipovich AH, Imashuku S, et al. $\mathrm{HLH}$-2004: Diagnostic and therapeutic guidelines for hemophagocytic lymphohistiocytosis. Pediatr Blood Cancer. 2007;48(2):124-31. Available from: http://www.ncbi.nlm.nih.gov/pubmed/16937360. Accessed 11 Oct 2016.

4. Wong KF, Hui PK, Chan JK, Chan YW, Ha SY. The acute lupus hemophagocytic syndrome. Ann Intern Med. 1991;114(5):387-90. Available from: http://www. ncbi.nlm.nih.gov/pubmed/1992881. Accessed 12 Oct 2016.

5. Takahashi K, Kumakura S, Ishikura H, Murakawa Y, Yamauchi Y, Kobayashi S. Reactive hemophagocytosis in systemic lupus erythematosus. Intern Med. 1998;37(6):550-3. Available from: http://www.ncbi.nlm.nih.gov/pubmed/ 9678692. Accessed 12 Oct 2016.

6. Fukaya S, Yasuda S, Hashimoto T, Oku K, Kataoka H, Horita T, et al. Clinical features of haemophagocytic syndrome in patients with systemic autoimmune diseases: analysis of 30 cases. Rheumatology (Oxford). 2008; 47(11):1686-91. Available from: http://www.ncbi.nlm.nih.gov/pubmed/ 18782855. Accessed 12 Oct 2016.

7. Jordan MB, Filipovich AH. Hematopoietic cell transplantation for hemophagocytic lymphohistiocytosis: a journey of a thousand miles begins with a single (big) step. Bone Marrow Transplant. 2008:42(7):433-7. Available from: http://www.ncbi. nlm.nih.gov/pubmed/18679369. Accessed 24 Oct 2016

8. Schram AM, Berliner N. How I treat hemophagocytic lymphohistiocytosis in the adult patient. Blood. 2015:125(19):2908-14

9. George MR. Hemophagocytic lymphohistiocytosis: review of etiologies and management. J Blood Med. 2014;5:69-86. Available from: http://www.ncbi. nlm.nih.gov/pubmed/24966707. Accessed 12 Jan 2017.

10. Petri M, Orbai A-M, Alarcón GS, Gordon C, Merrill JT, Fortin PR, et al. Derivation and validation of the Systemic Lupus International Collaborating Clinics classification criteria for systemic lupus erythematosus. Arthritis Rheum. 2012;64(8):2677-86. Available from: http://www.ncbi.nlm.nih.gov/ pubmed/22553077. Accessed 14 Nov 2016.

11. Noone P, Rogers BT. Pneumonia caused by coliforms and Pseudomonas aeruginosa. J Clin Pathol. 1976;29(7):652-6. Available from: http://www.ncbi. nlm.nih.gov/pubmed/824327. Accessed 8 Jan 2017.

12. Paul Forgacs. Problems in Respiratory Medicine - Paul Forgacs - Google Books [Internet]. MTP Press Limited. 1981. p. 41. Available from: https:// books.google.lk/books?id=swNMAQAAIAAJ\&source=gbs_book_other_ versions. Accessed 8 Jan 2017.

13. Wilkinson PJ, Ball AJ, Doran J, Gillespie WA, Orton VS. Routine laboratory assessment of postoperative chest infection: a prospective study. J Clin Pathol. 1977;30(5):417-20. Available from: http://www.ncbi.nlm.nih.gov/ pubmed/325018. Accessed 8 Jan 2017.

14. Tomita H, Yamada M, Sekigawa I, Yoshiike T, lida N, Hashimoto H. Systemic lupus erythematosus-like autoimmune abnormalities induced by bacterial infection. Clin Exp Rheumatol. 2003;21(4):497-9. Available from: http://www. ncbi.n/m.nih.gov/pubmed/12942705. Accessed 17 Feb 2017. 
15. Kasapcopur O, Ergul Y, Kutlug S, Candan C, Camcioglu Y, Arisoy N. Systemic lupus erythematosus due to Epstein-Barr virus or Epstein-Barr virus infection provocating acute exacerbation of systemic lupus erythematosus? Rheumatol Int. 2006;26(8):765-7. Available from: http://www.ncbi.nlm.nih. gov/pubmed/16200398. Accessed 22 Feb 2017.

16. Marcos M, Fernández C, Soriano À, Marco F, Martínez J, Almela M, et al. Epidemiology and clinical outcomes of bloodstream infections among lupus patients. Lupus. 2011;20(9):965-71. Available from: http://www.ncbi. nlm.nih.gov/pubmed/21659421. Accessed 17 Feb 2017.

17. Bosch $X$, Guilabert A, Pallarés L, Cervera R, Ramos-Casals M, Bové A, et al. Infections in systemic lupus erythematosus: a prospective and controlled study of 110 patients. Lupus. 2006;15(9):584-9. Available from: http://www. ncbi.nlm.nih.gov/pubmed/17080913. Accessed 21 Feb 2017.

18. Tsokos GC. Systemic Lupus Erythematosus. N Engl J Med. 2011;365(22): 2110-21. Available from: http://www.ncbi.nlm.nih.gov/pubmed/22129255. Accessed 21 Feb 2017.

Submit your next manuscript to BioMed Central and we will help you at every step:

- We accept pre-submission inquiries

- Our selector tool helps you to find the most relevant journal

- We provide round the clock customer support

- Convenient online submission

- Thorough peer review

- Inclusion in PubMed and all major indexing services

- Maximum visibility for your research

Submit your manuscript at www.biomedcentral.com/submit
Biomed Central 